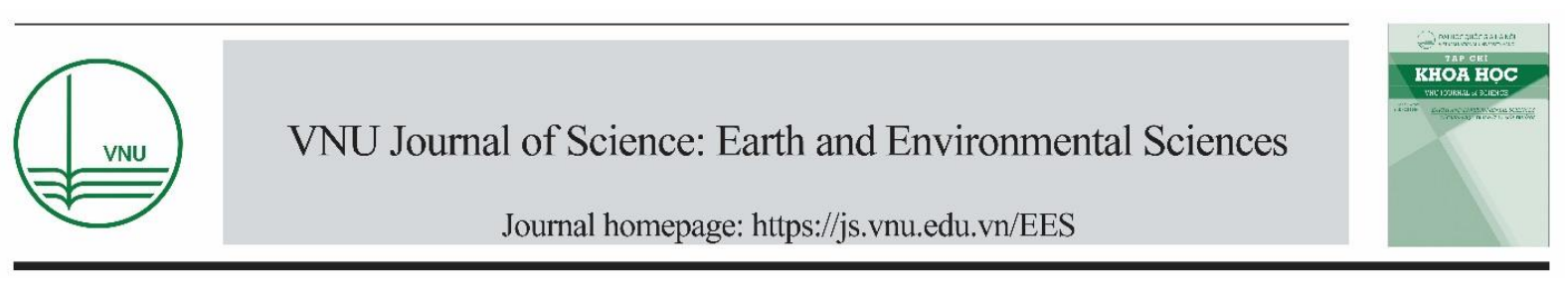

Original Article

\title{
Urban Bare Land Classification Using NDBaI Index Based on Combination of Sentinel 2 MSI and Landsat 8 Multiresolution Images
}

\author{
Trinh Le Hung* \\ Military Technical Academy, 236 Hoang Quoc Viet, Hanoi, Vietnam \\ Received 03 January 2020 \\ Revised 08 February 2020; Accepted 11 February 2020
}

\begin{abstract}
The classification of urban land cover/land use is a difficult task due to the complexity in the structure of the urban surface. This paper presents the method of combining of Sentinel 2 MSI and Landsat 8 multi-resolution satellite image data for urban bare land classification based on NDBaI index. Two images of Sentinel 2 and Landsat 8 acquired closely together, were used to calculate the NDBaI index, in which sortware infrared band (band 11) of Sentinel 2 MSI image and thermal infrared band (band 10) of Landsat 8 image were used to improve the spatial resolution of NDBaI index. The results obtained from two experimental areas showed that, the total accuracy of classifying bare land from the NDBaI index which calculated by the proposed method increased by about $6 \%$ compared to the method using the NDBaI index, which is calculated using only Landsat 8 data. The results obtained in this study contribute to improving the efficiency of using free remote sensing data in urban land cover/land use classification.
\end{abstract}

Keywords: bare land, classification, urban indices, NDBaI, Sentinel 2 MSI, Landsat 8.

\footnotetext{
* Corresponding author.

E-mail address: trinhlehung125@gmail.com

https://doi.org/10.25073/2588-1094/vnuees.4537
} 


\title{
Phân loại đất trống đô thị bằng chỉ số NDBaI trên cơ sở kết hợp ảnh vệ tinh đa độ phân giải Sentinel 2 MSI và Landsat 8
}

\author{
Trịnh Lê Hùng*
}

Học viện Kỹ thuật Quân sư, 236 Hoàng Quốc Việt, Hà Nội, Việt Nam

Nhận ngày 03 tháng 01 năm 2020

Chỉnh sửa ngày 08 tháng 02 năm 2020; Chấp nhận đăng ngày 11 tháng 02 năm 2020

\begin{abstract}
Tóm tắt: Phân loại lớp phủ/sử dụng đất khu vực đô thị là một công việc khó khăn do sự phức tạp trong cấu trúc của bề mặt đô thị. Bài báo này trình bày phương pháp kết hợp dữ liệu ảnh vệ tinh đa độ phân giải Sentinel 2 MSI và Landsat 8 trong phân loại đất trống trên cơ sở chỉ số NDBaI. 02 cảnh ảnh Sentinel 2 MSI và Landsat 8 chụp sát thời điểm với nhau được sử dụng để tính chỉ số $\mathrm{NDBaI}$, trong đó sử dụng kênh hồng ngoại giữa (kênh 11) ảnh Sentinel 2 MSI và kênh hồng ngoại nhiệt (kênh 10) ảnh Landsat 8 , giúp nâng cao độ phân giải không gian của chỉ số NDBaI. Kết quả nhận được cho thấy, tại 02 khu vực thử nghiệm, độ chính xác khi phân loại đất trống từ chỉ số NDBaI bằng phương pháp đề xuất tăng lên khoảng $6 \%$ so với phương pháp sử dụng chỉ số $\mathrm{NDBaI}$ tính từ ảnh vệ tinh Landsat 8 . Kết quả nhận được trong nghiên cứu góp phần nâng cao hiệu quả sử dụng các dữ liệu viễn thám miễn phí trong phân loại lớp phủ/sử dụng đất khu vực đô thị.
\end{abstract}

Từ khoá: đất trống đô thị, phân loại, chỉ số đất đô thị, NDBaI, Sentinel 2 MSI, Landsat 8.

\section{Mở đầu}

Phân loại lớp phủ/sử dụng đất khu vực đô thị là một vấn đề có tính thực tiễn hiện nay, phục vụ công tác quy hoạch và phát triển bền vững các đô thị. Từ những thập niên cuối thế kỷ XX, nhiều nghiên cứu đã sử dụng dữ liệu viễn thám trong phân loại lớp phủ/sử dụng đất khu vực đô thị. Thời gian đầu, phương pháp phân loại tự động (có giám sát và không giám sát) được sử dụng để chiết tách thông tin đất trống/đất xây dựng. Xu (2002) [1] đã sử dụng phương pháp phân loại có giám sát kết hợp với phân tích đặc trưng phản xạ phổ của các lớp sử dụng đất trong phân loại đất đô thị ở thành phố Fuqing, đông nam Trung Quốc. Nghiên cứu của Masek và CS (2000) [2] sử dụng phương pháp phân loại không giám sát cùng với chỉ số thực vật NDVI (Normalized
Difference Vegetation Index) để thành lập bản đồ sử dụng đất đô thị ở Washington (Mỹ). Guindon và $\mathrm{CS}$ (2004) [3] đề xuất phương pháp kết hợp kết quả phân loại không giám sát và phân mảnh (segmentation) để tăng độ chính xác cho các lớp sử dụng đất từ tư liệu ảnh vệ tinh Landsat. Xian và Crane (2005) [4] sử dụng thuật toán cây quyết định để thành lập bản đồ bề mặt không thấm ở khu vực đô thị. Nhìn chung, việc phân loại đất trống/đất xây dựng bằng phương pháp phân loại tự động gặp rất nhiều khó khăn do sự phức tạp của bề mặt đô thị, trong đó từng pixel có thể bao gồm nhiều đối tượng lớp phủ khác nhau.

Để khắc phục những hạn chế trên, một số nghiên cứu đã đề xuất sử dụng các chỉ số đất đô thị (urban indices) nhằm nâng cao độ chính xác khi phân loại lớp phủ/sử dụng đất khu vực đô thị.

\footnotetext{
* Tác giả liên hệ.

Địa chỉ email: trinhlehung125@gmail.com

https://doi.org/10.25073/2588-1094/vnuees.4537
} 
Ridd (1995) [5] đã mô hình hóa đô thị từ ba hợp phần (V-I-S): Cây xanh (V - vegetation), bề mặt không thấm (I - impervious surface) và đất ( $\mathrm{S}$ soil) nhằm đánh giá sự thay đổi của đô thị. Mô hình V-I-S đã được sử dụng rộng rãi trong các nghiên cứu về phân loại lớp phủ/sử dụng đất đô thị, tuy nhiên mô hình này cũng có nhược điểm khi không tính đến hợp phần nước [6]. Zha và CS (2003) [7] đã đề xuất chỉ số NDBI (Normalized Difference Built - up Index) trong phân loại đất xây dựng dựa trên cơ sở đất xây dựng có hệ số phản xạ ở dải sóng giữa hồng ngoại (MIR) cao hơn hẳn so với dải sóng cận hồng ngoại (NIR). Xu (2008) [8] đã đề xuất chỉ số đất xây dựng IBI (Index based Built-up Index) trong theo dõi quá trình đô thị hóa trên cơ sở 3 hợp phần: cây xanh (chỉ số SAVI - Soil Adjusted Vegetation Index), nước (chỉ số MNDWI Modified Normalized Difference Water Index) và đất xây dựng (chỉ số $\mathrm{NDBI}$ ). Chỉ số IBI cũng được sử dụng trong nghiên cứu của Nguyễn Hoàng Khánh Linh (2011) [9] khi phân loại đất xây dựng khu vực thành phố Huế. As-syakur và CS (2012) [10] đề xuất chỉ số EBBI (Enhanced Build - up and Bareness Index) trong theo dõi biến động đất đô thị ở Bali (Indonesia). Chỉ số EBBI được xây dựng trên cơ sở kết hợp các kênh hồng ngoại và hồng ngoại nhiệt ảnh vệ tinh Landsat. Chỉ số EBBI cũng được sử dụng trong nhiều nghiên cứu khác như của Rasul và $C S$ (2018) [11], Li và CS (2017) [12], Sekertekin và CS (2018) [13], Bramhe và CS (2018) [14],... nhằm phân loại đất xây dựng và đất trống khu vực đô thị. Gadal và Ouerghemmi (2019) [15] kết hợp sử dụng nhiều chỉ số khác nhau trong chiết tách thông tin lớp phủ/sử dụng đất khu vực Yakutsk (Liên bang Nga). Độ chính xác khi phân loại đất trống/đất đô thị trong các nghiên cứu này đạt khoảng trên $80 \%$.

Đất trống (bare land) là một hợp phần quan trọng của lớp phủ khu vực đô thị. Đặc trưng phản xạ phổ của đất trống không có nhiều khác biệt so với một số loại hình lớp phủ đô thị khác như đất xây dựng dẫn đến những khó khăn khi phân loại tự động từ ảnh vệ tinh. Các chỉ số đất đô thị như UI, EBBI cho phép phân loại cả đất trống và đất xây dựng, tuy nhiên độ chính xác thường không cao. Zhao và Chen (2004) [16] đã đề xuất chỉ số NDBaI (Normalized Difference Bareness Index) trên cơ sở kết hợp kênh hồng ngoại nhiệt và hồng ngoại giữa ảnh vệ tinh Landsat TM để phân loại riêng đất trống trên ảnh vệ tinh quang học. Một số nghiên cứu sau đó như Ghosh và CS (2018) [17], Firozijaei và CS (2019) [18], Mustafa và CS (2013) [19] cũng đã chứng minh tính hiệu quả khi phân loại đất trống bằng chỉ số $\mathrm{NDBaI}$ so với sử dụng các chỉ số đất đô thị khác. Mặc dù vậy, do độ phân giải không gian của ảnh Landsat không cao, việc sử dụng chỉ số NDBaI xác định từ ảnh Landsat trong phân loại đất trống ở các đô thị nhỏ gặp rất nhiều khó khăn. Điều này có thể khắc phục khi sử dụng ảnh vệ tinh đa độ phân giải chụp cùng hoặc sát thời điểm. Do biến động bề mặt lớp phủ trong thời gian rất ngắn (dưới 1 ngày) là không đáng kể, việc kết hợp các ảnh vệ tinh khác nhau khi tính chỉ số NDBaI là hoàn toàn phù hợp.

Nghiên cứu này trình bày kết quả phân loại đất trổng từ chỉ số $\mathrm{NDBaI}$ trên cơ sở kết hợp ảnh vệ tinh đa độ phân giải Sentinel 2 MSI và Landsat $8 \mathrm{OLI}$ chụp sát thời điểm với nhau. Kênh hồng ngoại nhiệt (kênh 10) ảnh vệ tinh Landsat 8 OLI và kênh hồng ngoại sóng ngắn (kênh 11) ảnh vệ tinh Sentinel 2 MSI được sử dụng để tính chỉ số NDBaI. Do kênh hồng ngoại sóng ngắn ảnh vệ tinh Sentinel 2 MSI có độ phân giải không gian $20 \mathrm{~m}$, việc kết hợp hai loại ảnh vệ tinh này giúp nâng cao độ phân giải không gian của chỉ số $\mathrm{NDBaI}$ lên thành $20 \mathrm{~m}$ và có thể áp dụng hiệu quả khi phân loại các khu vực có diện tích không lớn.

\section{Dữ liệu và phương pháp nghiên cứu}

\subsection{Dũ liêu viễn thám}

Dữ liệu viễn thám sử dụng trong nghiên cứu bao gồm 02 cảnh ảnh vệ tinh Landsat 8 và 02 cảnh ảnh Sentinel 2. Các ảnh Landsat 8 chụp ngày 02/07/2018 (khu vực thành phố Thanh Hóa, tỉnh Thanh Hóa) và 30/09/2019 (khu vực thành phố Thái Nguyên, tỉnh Thái Nguyên). 02 cảnh ảnh Sentinel 2 chụp ngày 03/07/2018 (khu vực thành phố Thanh Hóa, tỉnh Thanh Hóa) và 
01/10/2019 (khu vực thành phố Thái Nguyên, tỉnh Thái Nguyên). Đối với từng khu vực thử nghiệm, các ảnh vệ tinh Landsat 8 và Sentinel 2 MSI được lựa chọn chụp vào thời điểm sát nhau để hạn chế ảnh hưởng do sự khác biệt về thời gian gây nên.

Landsat 8 là thế hệ vệ tinh thứ 8 của chương trình Landsat (NASA, Mỹ), được phóng lên quỹ đạo vào ngày 11 tháng 02 năm 2013, sử dụng 2 bộ cảm biến: bộ cảm quang học OLI và bộ cảm hồng ngoại nhiệt TIRS. Ảnh Landsat 8 bao gồm 11 kênh, trong đó có 8 kênh đa phổ (độ phân giải không gian $30 \mathrm{~m}), 1$ kênh toàn sắc $(15 \mathrm{~m})$ và 2 kênh hồng ngoại nhiệt (100m). Chu kỳ chụp lặp lại tại một vị trí trên bề mặt Trái Đất của vệ tinh Landsat 8 là 16 ngày.

Vệ tinh Sentinel 2 MSI, bao gồm 2 vệ tinh có đặc điểm hoàn toàn giống nhau (Sentinel $2 \mathrm{~A}$ (2015) và Sentinel 2B (2017)) cung cấp ảnh ở 13 kênh phổ trong dải sóng nhìn thấy và hồng ngoại với chu kỳ cập nhật trong 5 ngày. Ảnh Sentinel 2 MSI có độ phân giải không gian từ $10-60 \mathrm{~m}$, trong đó kênh hồng ngoại giữa (kênh 11) có độ phân giải không gian $20 \mathrm{~m}$.

Do tần suất chụp gần sát thời điểm (thậm chí trùng nhau) của vệ tinh Landsat 8 và Sentinel 2 là rất lớn, hơn nữa, cả hai vệ tinh này đều cung cấp dữ liệu miễn phí, việc kết hợp ảnh Sentinel và Landsat 8 trong nâng cao hiệu quả chiết tách thông tin bề mặt Trái Đất là hoàn toàn khả thi. Mặt khác, đặc điểm các kênh phổ ảnh vệ tinh Sentinel 2 MSI và Landsat 8 cũng có nhiều tương đồng, từ bước sóng trung tâm đến độ rộng kênh phổ (bandwidth) (Pahlevan và CS (2017) - Bảng 1) [20]. Điều này cũng giúp chứng minh phương án kết hợp ảnh Sentinel 2 MSI và Landsat 8 trong tính chỉ số NDBaI phục vụ phân loại đất trống khu vực đô thị là phù hợp.

Bảng 1. So sánh đặc điểm các kênh ảnh vệ tinh Sentinel 2 MSI và Landsat 8 OLI [20]

\begin{tabular}{|c|c|c|c|c|c|c|c|c|c|c|c|}
\hline \multicolumn{12}{|c|}{ Bước sóng trung tâm (nm) } \\
\hline MSI & 444 & 497 & 560 & 664 & 704 & 740 & 783 & 843 & 865 & 1613 & 2190 \\
\hline OLI & 443 & 482 & 561 & 665 & NA & NA & NA & NA & 865 & 1609 & 2201 \\
\hline \multicolumn{12}{|c|}{ Độ rộng kênh phồ (nm) } \\
\hline MSI & 20 & 55 & 35 & 30 & 15 & 15 & 15 & 115 & 20 & 90 & 175 \\
\hline OLI & 20 & 65 & 60 & 40 & NA & NA & NA & NA & 30 & 85 & 190 \\
\hline \multicolumn{12}{|c|}{ Độ phân giải không gian (m) } \\
\hline MSI & 60 & 10 & 10 & 10 & 20 & 20 & 20 & 10 & 20 & 20 & 20 \\
\hline OLI & 30 & 30 & 30 & 30 & NA & NA & NA & NA & 30 & 30 & 30 \\
\hline
\end{tabular}

\subsection{Phưong pháp nghiên cứu}

Trong nghiên cứu này, chỉ số NDBaI được sử dụng để phân loại đất trống khu vực đô thị. Chỉ số NDBaI được Zhao và Chen (2004) đề xuất trên cơ sở ảnh vệ tinh Landsat TM theo công thức sau:

$$
N D B a I=\frac{\text { Band } 5-\text { Band } 6}{\text { Band } 5+\text { Band } 6}
$$

Trong đó Band 5 và Band6 tương ứng là giá trị số nguyên ở các kênh hồng ngoại giữa và hồng ngoại nhiệt ảnh Landsat TM.

Khi kết hợp ảnh vệ tinh đa độ phân giải Sentinel 2 MSI và Landsat 8 , chỉ số NDBaI xác định như sau:

$$
N D B a I=\frac{\text { Band } 11_{\text {Sentinel } 2}-\text { Band } 10_{\text {Landsat } 8}}{\text { Band } 11_{\text {Sentinel } 2}+\text { Band } 10_{\text {Landsat } 8}}
$$

Trong đó, Band11 là kênh hồng ngoại sóng ngắn ảnh vệ tinh Sentinel 2 MSI, Band10 là kênh hồng ngoại nhiệt ảnh Landsat 8.

Sau khi tính chỉ số NDBaI, phương pháp phân ngưỡng (threshold) được sử dụng để phân loại đất trống. Quy trình phân loại đất trống khu vực đô thị bằng chỉ số NDBaI trên cơ sở kết hợp ảnh vệ tinh đa độ phân giải Sentinel 2 và Landsat 8 được mô tả trên Hình 1. 


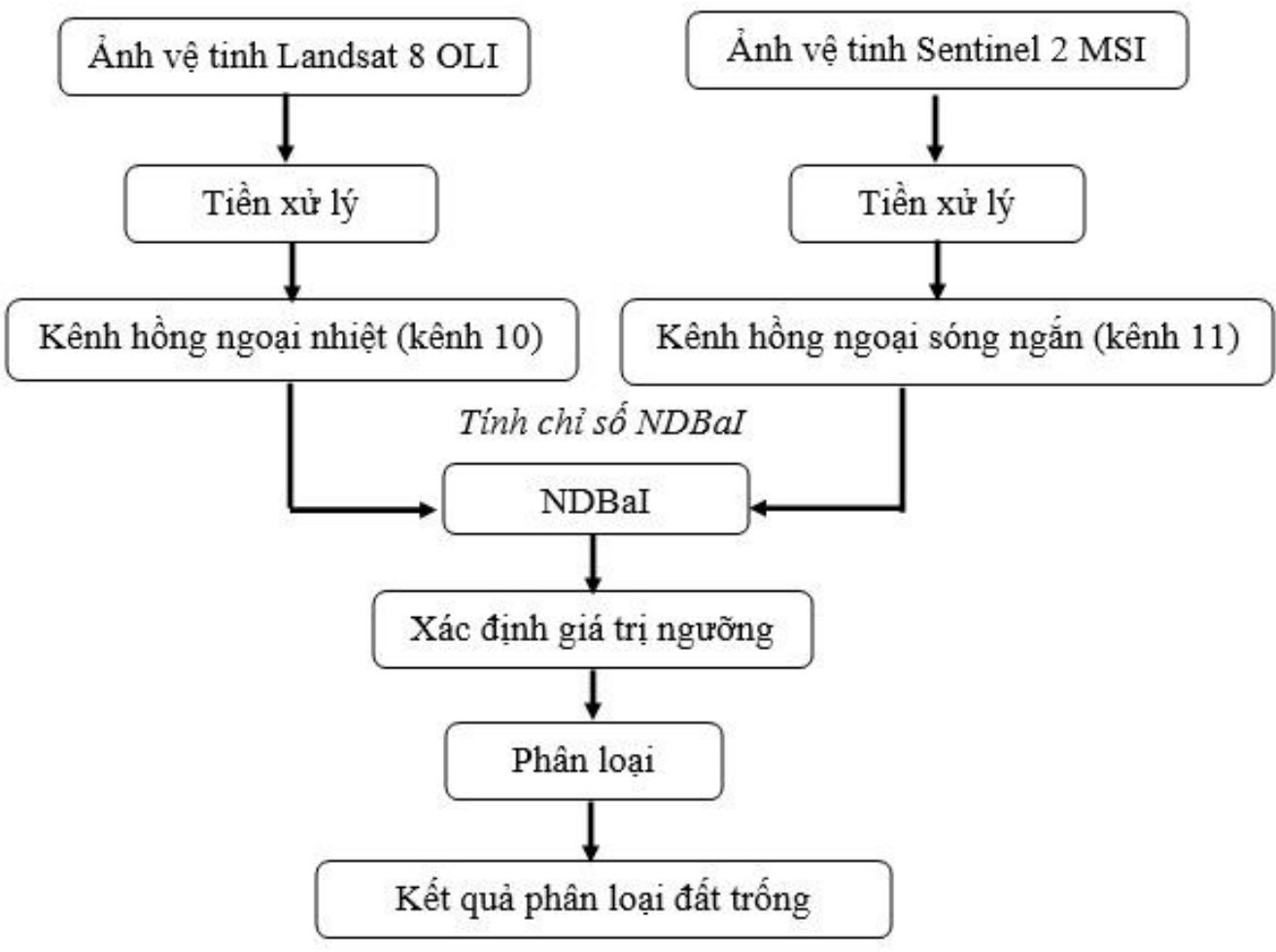

Hình 1. Sơ đồ phương pháp phân loại đất trống từ chỉ số NDBaI trên cơ sở kết hợp ảnh vệ tinh Landsat 8 và Sentinel 2 MSI

\section{Kết quả và thảo luận}

\subsection{Khu vục thư nghiệm 1}

Khu vực thử nghiệm 1 được lựa chọn là thành phố Thanh Hóa, tỉnh Thanh Hóa. Đây là một đô thị lớn ở Bắc Trung Bộ, có tốc độ đô thị hóa nhanh. Trong thử nghiệm này, ảnh vệ tinh Sentinel 2A chụp ngày $03 / 07 / 2018$ (Hình 2 ) và Landsat 8 chụp ngày 02/07/2018 (Hình 3) được sử dụng để tính chỉ số NDBaI theo công thức (2). Kết quả xác định chỉ số NDBaI trên cơ sở kết hợp ảnh Sentinel 2 MSI và Landsat 8 khu vực thành phố Thanh Hóa được trình bày trên Hình 4, trong đó các pixel có màu trắng sáng đại diện cho các khu vực đất trống. Các pixel màu xám thể hiện khu vực đất xây dựng và các pixel màu tối thể hiện khu vực mặt nước hoặc có thực vật che phủ.

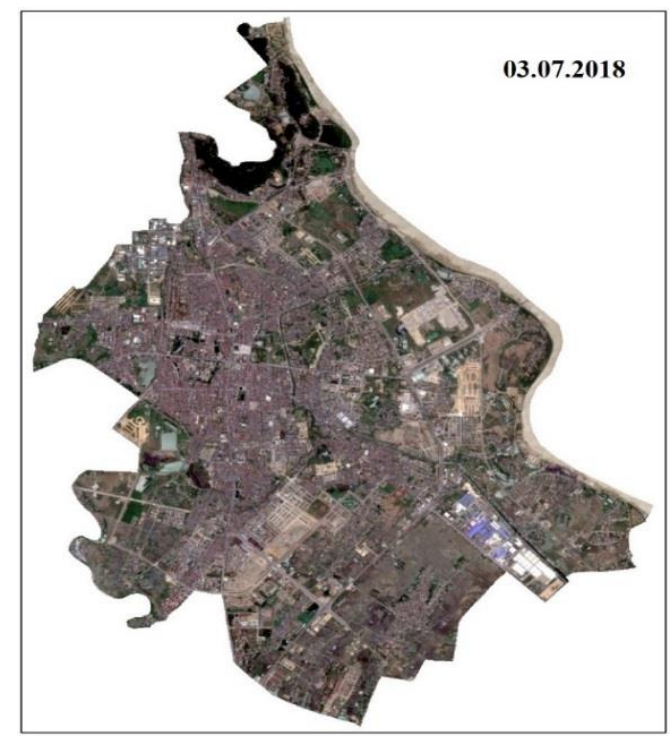

Hình 2. Ảnh vệ tinh Sentinel 2 ngày 03/07/2018 khu vực thành phố Thanh Hóa. 


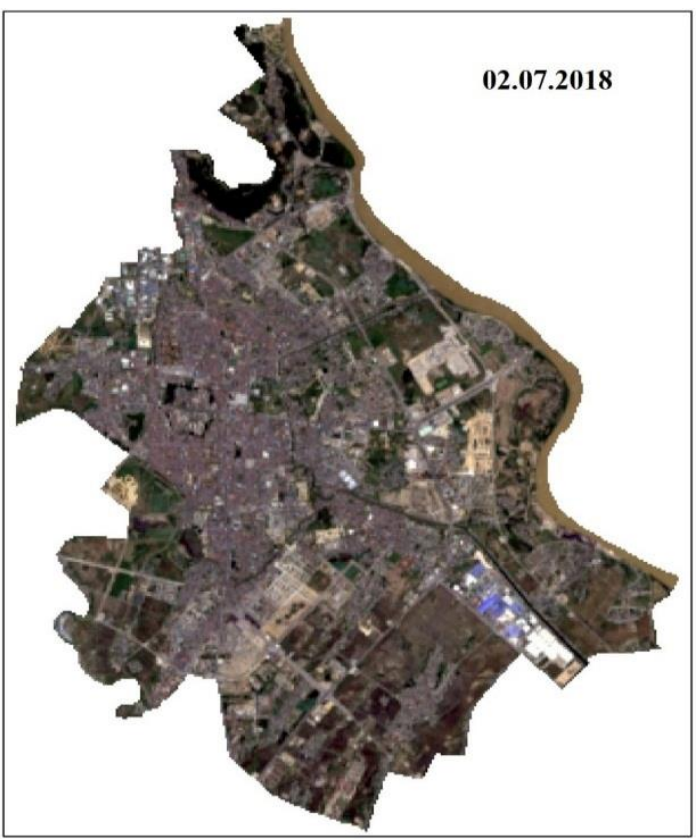

Hình 3. Ảnh vệ tinh Landsat 8 ngày 02/07/2018 khu vực thành phố Thanh Hóa.

Để so sánh, trong nghiên cứu cũng tiến hành xác định chỉ số NDBaI từ riêng ảnh Landsat 8 theo công thức (1).

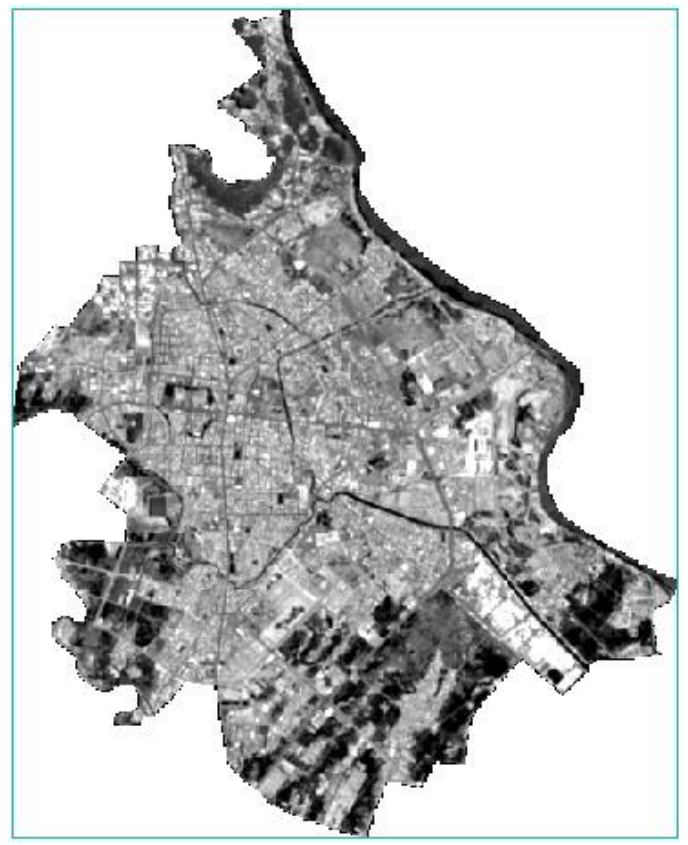

Hình 4. Chỉ số NDBaI khu vực thành phố Thanh Hóa xác định từ ảnh Sentinel 2 MSI và Landsat 8 .
Kết quả tính chỉ số NDBaI từ ảnh Landsat 8 chụp ngày 02/07/2018 khu vực thành phố Thanh Hóa, tỉnh Thanh Hóa được trình bày trên Hình 5.

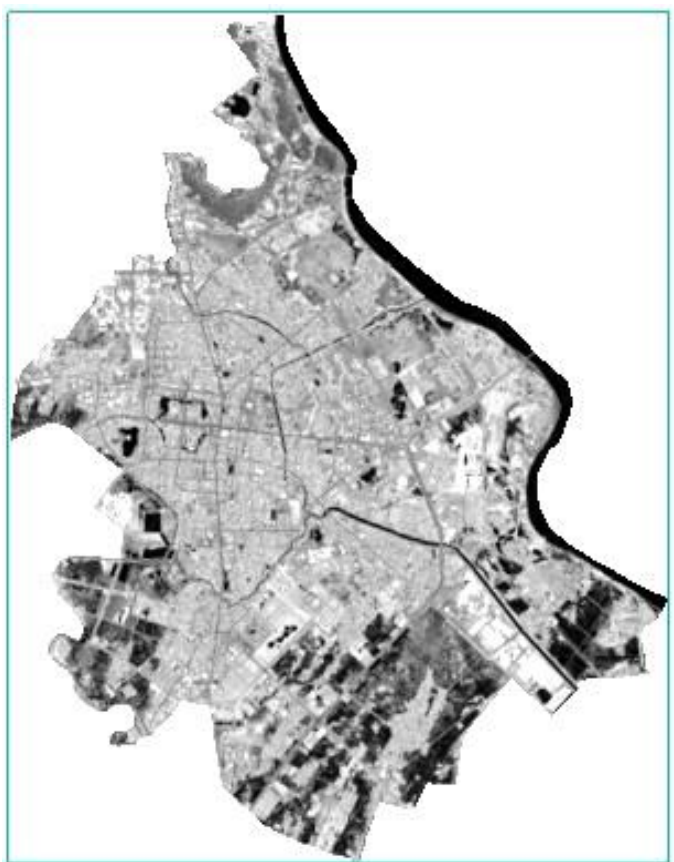

Hình 5. Chỉ số NDBaI khu vực thành phố Thanh Hóa xác định từ ảnh Landsat 8.

Kết quả phân loại đất trống khu vực thành phố Thanh Hóa, tỉnh Thanh Hóa bằng phương pháp phân ngưỡng từ chỉ số NDBaI xác định từ ảnh vệ tinh đa độ phân giải Sentinel 2 và Landsat 8 được trình bày trên Hình 6 . Trong thử nghiệm này, giá trị ngưỡng $(-0,80822)$ được lấy bằng phương pháp chuyên gia trên cơ sở phân tích lược đồ histogram của ảnh chỉ số NDBaI. Quá trình thực hiện được tiến hành trên phần mềm xử lý ảnh ENVI 5.2.

Để so sánh, trong nghiên cứu cũng tiến hành phân loại đất trống từ chỉ số $\mathrm{NDBaI}$ tính từ ảnh vệ tinh Landsat 8 ngày 02/07/2018. Kết quả phân loại đất trống khu vực thành phố Thanh Hóa từ chỉ số NDBaI sử dụng ảnh Landsat 8 được thể hiện trên Hình 7. Trong ví dụ này, giá trị ngưỡng đối với đất trống được lấy bằng $(-0,17887)$ thông qua phân tích histogram. 


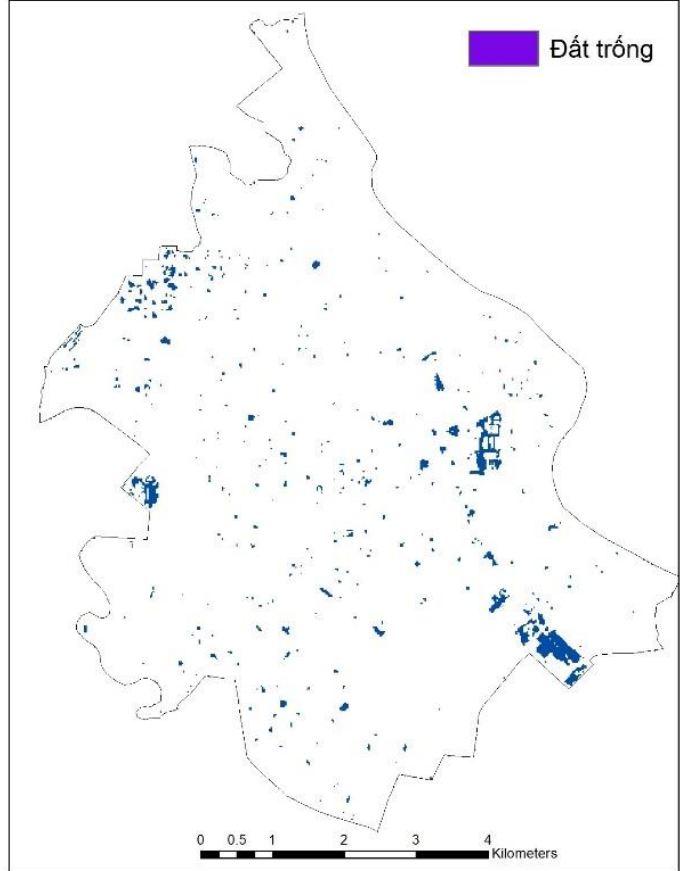

Hình 6. Kết quả phân loại đất trống khu vực thành phố Thanh Hóa bằng chỉ số $\mathrm{NDBaI}$ xác định từ ảnh Sentinel 2 MSI và Landsat 8.

Để đánh giá độ chính xác kết quả phân loại, 250 điểm ngẫu nhiên từ ảnh phân loại đất trống đô thị khu vực thành phố Thanh Hóa được so sánh với ảnh vệ tinh độ phân giải cao thu thập từ Google Earth. Kết quả cho thấy, độ chính xác tổng thể khi phân loại đất trống từ chỉ số $\mathrm{NDBaI}$ khi kết hợp ảnh vệ tinh Landsat 8 và Sentinel 2 MSI đạt 92,80\%, chỉ số Kappa đạt 0,916. Đối với phương án chỉ sử dụng ảnh vệ tinh Landsat 8 , giá trị độ chính xác chung và chỉ số Kappa đạt lần lượt là $86,40 \%$ và 0.852 . Như vậy, việc kết hợp ảnh vệ tinh đa độ phân giải Sentinel 2 và Landsat 8 trong phân loại đất trống bằng chỉ số $\mathrm{NDBaI}$ cho phép nâng cao độ chính xác kết quả phân loại lên khoảng $6 \%$.

Bên cạnh đó, trong nghiên cứu cũng tiến hành phân loại đất trống bằng phương pháp phân loại tự động có giám sát sử dụng thuật toán xác suất cực đại (maximum likelihood) từ ảnh Sentinel 2A chụp ngày 03/07/2018. Kết quả nhận được cho thây, độ chính xác tổng thể và giá trị chỉ số Kappa khi phân loại đất trống bằng phương pháp này đạt $82,17 \%$ và 0,797 .

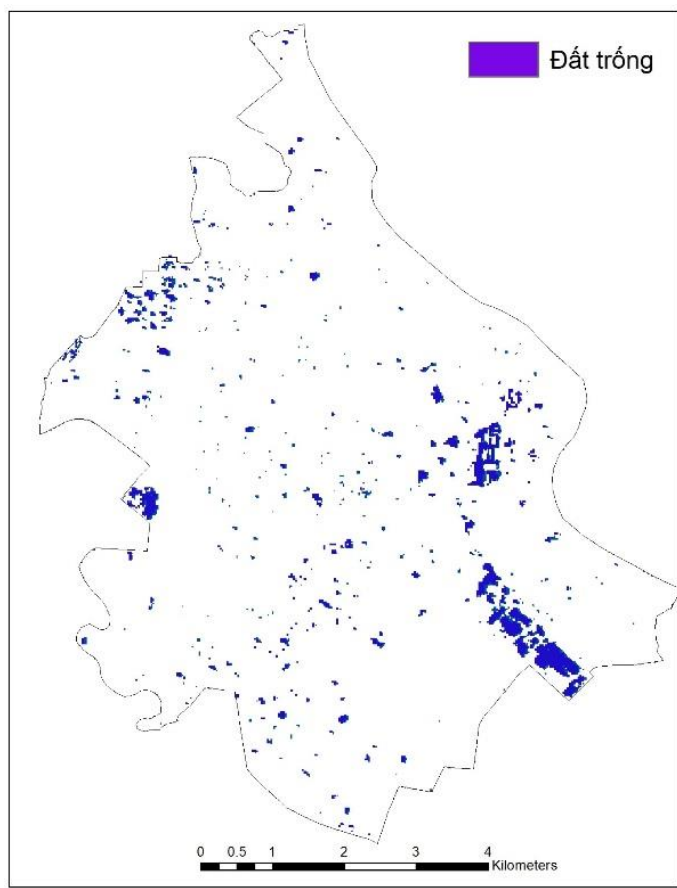

Hình 7. Kết quả phân loại đất trống khu vực thành phố Thanh Hóa bằng chỉ số NDBaI xác định từ ảnh Landsat 8.

\subsection{Khu vục thư nghiệm 2}

Khu vực thử nghiệm 2 được lựa chọn là thành phố Thái Nguyên, tỉnh Thái Nguyên. Đây là một đô thị có diện tích không lớn, việc phân loại lớp phủ/sử dụng đất bằng ảnh vệ tinh có độ phân giải $30 \mathrm{~m}$ như ảnh Landsat 8 gặp rất nhiều khó khăn.

Trong ví dụ này, ảnh vệ tinh Sentinel 2A chụp ngày 01/10/2019 (Hình 8) và ảnh Landsat 8 chụp ngày 30/9/2019 (Hình 9) được sử dụng để tính chỉ số NDBaI, sau đó phân loại đất trống bằng phương pháp phân ngưỡng như đối với khu vực thử nghiệm 1 . Giá trị ngưỡng cũng được lựa chọn trên cơ sở phân tích histogram ảnh chỉ số NDBaI. Đối với chỉ số NDBaI xác định từ việc kết hợp ảnh Sentinel 2 MSI và Landsat 8 , giá trị ngưỡng được lấy là $-0,81170$, trong khi đó, với chỉ số NDBaI tính từ riêng ảnh Landsat 8 , giá trị ngưỡng được lựa chọn là -0,21455. 


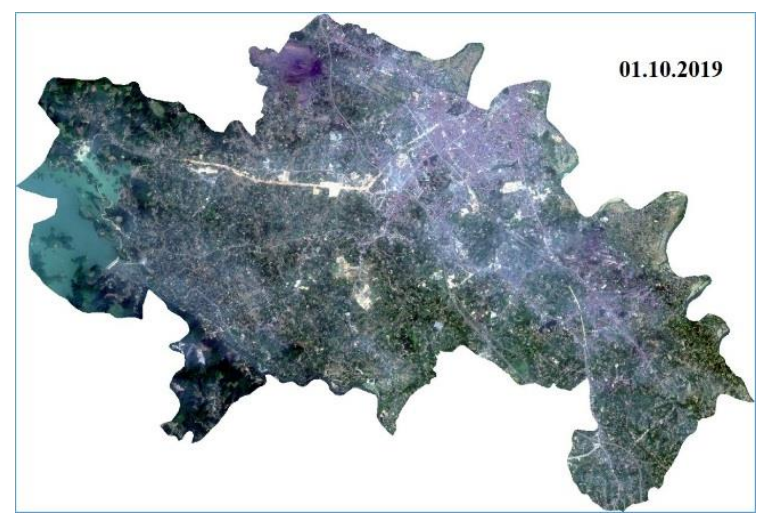

Hình 8. Ảnh vệ tinh Sentinel 2A ngày 01/10/2019 khu vực thành phố Thái Nguyên, tỉnh Thái Nguyên.

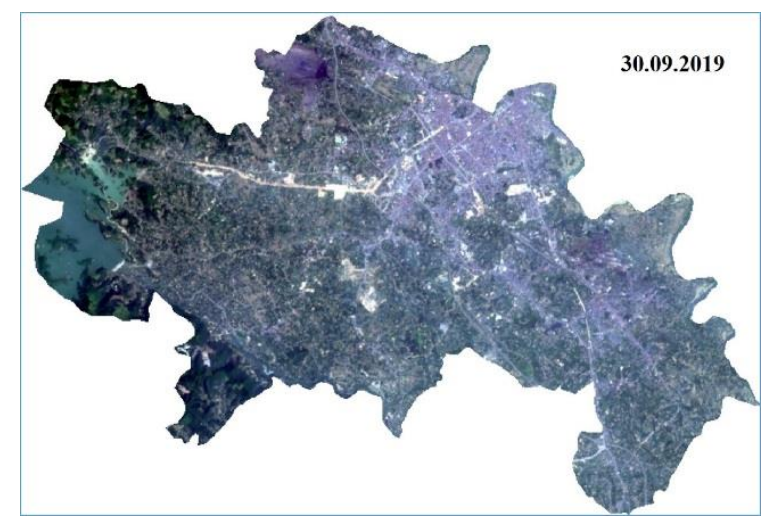

Hình 9. Ảnh vệ tinh Landsat 8 ngày 30/09/2019 khu vực thành phố Thái Nguyên, tỉnh Thái Nguyên.

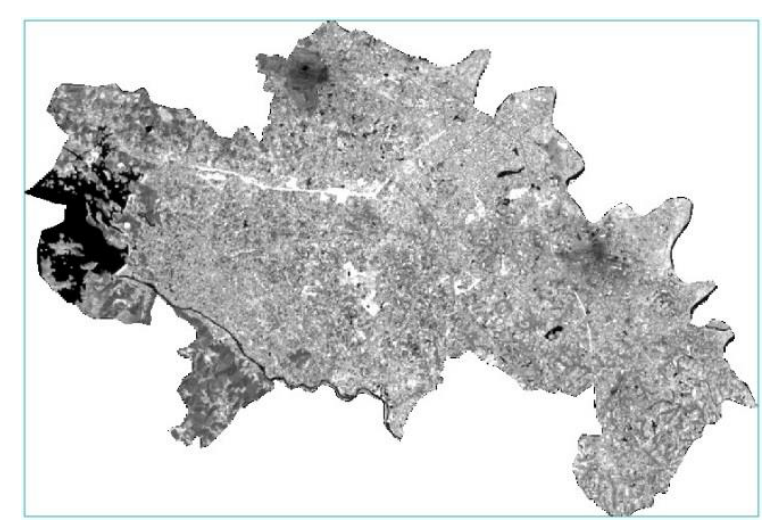

Hình 10. Chỉ số NDBaI khu vực thành phố Thái Nguyên xác định từ ảnh vệ tinh Sentinel 2 MSI và Landsat 8.

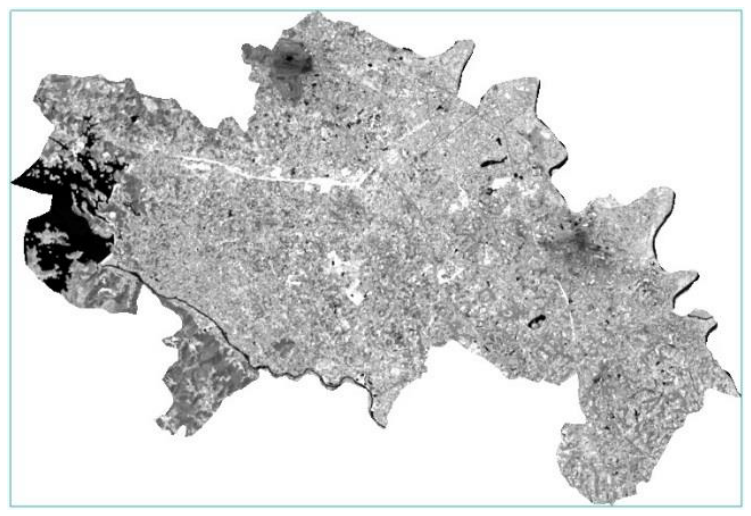

Hình 11. Chỉ số NDBaI khu vực thành phố Thái Nguyên xác định từ ảnh vệ tinh Landsat 8.

Kết quả xác định chỉ số NDBaI bằng hai phương án trên được trình bày trên các Hình 10 và 11 , trong đó các pixel màu sáng trắng thể hiện các khu vực đất trống đô thị.

Trên Hình 12 và 13 thể hiện kết quả phân loại đất trống khu vực thành phố Thanh Hóa từ chỉ số NDBaI xác định bằng cách kết hợp ảnh vệ tinh đa độ phân giải Setinel 2 và Landsat 8 và phương án chỉ sử dụng riêng ảnh Landsat 8 . Để đánh giá độ chính xác kết quả phân loại, trong ví dụ này cũng sử dụng 250 điểm kiểm tra ngẫu nhiên lấy từ kết quả phân loại, sau đó so sánh với ảnh vệ tinh độ phân giải cao thu thập từ Google Earth. Kết quả cho thấy, khi phân loại đất trống từ chỉ số $\mathrm{NDBaI}$ bằng cách kết hợp ảnh vệ tinh Landsat 8 và Sentinel 2 MSI, độ chính xác tổng thể đạt $91,60 \%$, chỉ số Kappa đạt 0,904. Đối với phương án chỉ sử dụng ảnh vệ tinh Landsat 8 , giá trị độ chính xác chung và chỉ số Kappa đạt lần lượt là $86,00 \%$ và 0,848 . Như vậy, cũng giống như với thử nghiệm 1 , phương pháp kết hợp ảnh vệ tinh đa độ phân giải Sentinel 2 MSI và Landsat 8 khi phân loại đất trống đô thị bằng chỉ số $\mathrm{NDBaI}$ cho phép nâng cao độ chính xác lên khoảng gần $6 \%$ so với phương pháp chỉ sử dụng riêng ảnh Landsat 8 . Đối với kết quả phân loại đất trống bằng phương pháp phân loại xác suất cực đại, độ chính xác tổng thể chỉ đạt $82,52 \%$, giá trị chỉ số Kappa là 0,814 . 


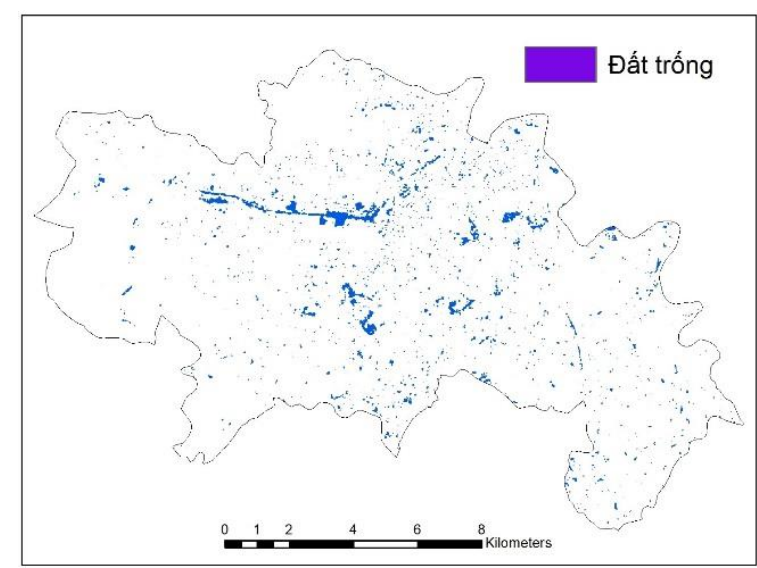

Hình 12. Kết quả phân loại đất trống khu vực thành phố Thái Nguyên bằng chỉ số NDBaI xác định từ ảnh Sentinel 2 MSI và Landsat 8.

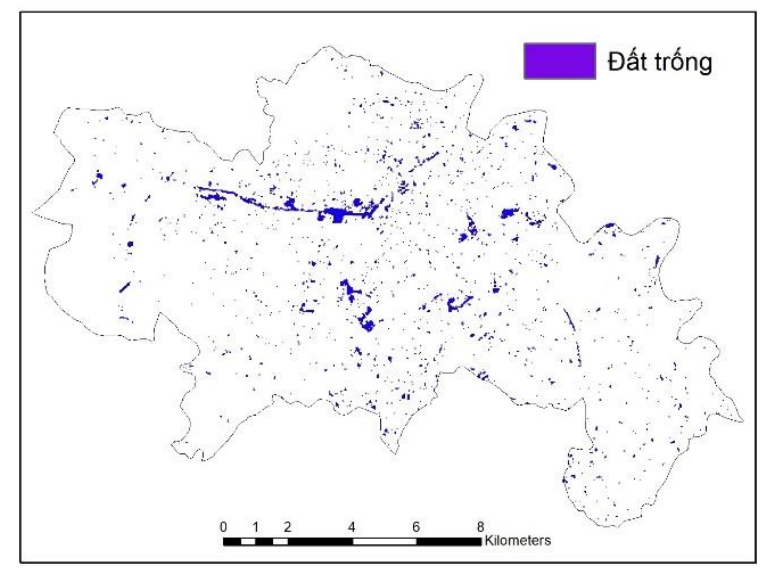

Hình 13. Kết quả phân loại đất trống khu vực thành phố Thái Nguyên bằng chỉ số NDBaI xác định từ ảnh Landsat 8.

\subsection{Một số nhận xét}

Từ 02 thử nghiệm trên cho thấy, việc kết hợp ảnh vệ tinh đa độ phân giải Sentinel 2 MSI và Landsat 8 chụp sát thời điểm nhau để tính chỉ số NDBaI nhằm phân loại đất trống đô thị cho phép nâng cao đáng kể độ chính xác (khoảng 6\%) so với phương pháp chỉ sử dụng ảnh vệ tinh Landsat 8. Giá trị chỉ số Kappa khi phân loại đất trống đô thị trong trường hợp sử dụng kết hợp ảnh vệ tinh Sentinel 2 MSI và Landsat 8 cũng cao hơn so với trường hợp chỉ sử dụng ảnh Landsat 8 . Hơn nữa, độ phân giải không gian của chỉ số NDBaI khi kết hợp ảnh vệ tinh Sentinel 2 MSI và Landsat 8 cũng đạt $20 \mathrm{~m}$ so với $30 \mathrm{~m}$ khi chỉ sử dụng ảnh Landsat 8 . Điều này làm giúp tăng tính hiệu quả khi áp dụng chỉ số NDBaI trong phân loại đất trống ở những đô thị có diện tích không lớn.

Bên cạnh đó, từ các thử nghiệm tại thành phố Thanh Hóa (tỉnh Thanh Hóa) và thành phố Thái Nguyên (tỉnh Thái Nguyên) cũng cho thấy, việc sử dụng chỉ số $\mathrm{NDBaI}$ trong phân loại đất trống đô thị cũng có những hạn chế nhất định khi phân loại nhầm lẫn những khu công nghiệp được lợp mái tôn, nhất là mái tôn màu trắng vào lớp đất trống (Bảng 2). Điều này có thể giải thích do phản xạ phổ của các khu công nghiệp được lợp tôn trắng rất giống với các khu vực đất trống, trong khi độ phân giải phổ của ảnh Sentinel 2 MSI và Landsat 8 không cho phép phân biệt rõ ràng hai đối tượng này. Để nâng cao độ chính xác, cần thiết phải tiến hành các công đoạn sau phân loại (post-classification) nhằm loại bỏ những khu vực bị phân loại nhầm lẫn.

\section{Kết luận}

Sử dụng các chỉ số đất đô thị xác định từ dữ liệu viễn thám trong phân loại đất trống là một tiếp cận phù hợp, giúp tiết kiệm thời gian cũng như nâng cao độ chính xác so với các phương pháp phân loại truyền thống.

Kết quả phân loại đất trống từ chỉ số NBDaI ở $02 \mathrm{khu}$ vực thử nghiệm, bao gồm thành phố Thanh Hóa (tỉnh Thanh Hóa) và thành phố Thái Nguyên (tỉnh Thái Nguyên) cho thấy, phương pháp kết hợp ảnh vệ tinh đa độ phân giải Sentinel 2 MSI và Landsat 8 giúp nâng cao độ chính xác tổng thể lên khoảng $6 \%$ so với phương pháp chỉ sử dụng ảnh Landsat 8 . Hơn nữa, độ phân giải không gian của chỉ số $\mathrm{NDBaI}$ khi tính từ ảnh Sentinel 2 và Landsat 8 cũng được nâng lên thành $20 \mathrm{~m}$, so với $30 \mathrm{~m}$ khi sử dụng riêng ảnh Landsat 8 . Điều này có ý nghĩa quan trọng trong trường hợp phân loại đất trống ở những khu vực đô thị có diện tích không lớn.

Từ kết quả nhận được trong nghiên cứu cũng cho thấy sự cần thiết phải tiển hành các công đoạn sau phân loại nhằm loại bỏ các sai số khi 
phân loại nhầm lẫn đất trống đô thị với các khu vực được lợp mái tôn, đặc biệt là mái tôn màu trắng. Điều này giúp nâng cao độ chính xác cũng như hiệu quả sử dụng các dữ liệu viễn thám miễn phí như ảnh Sentinel 2 MSI và Landsat 8 trong phân loại lớp phủ/sử dụng đất khu vực đô thị.

Bảng 2. Một số vị trí bị phân loại nhầm lẫn khi phân loại đất trống đô thị bằng chỉ số NBDaI

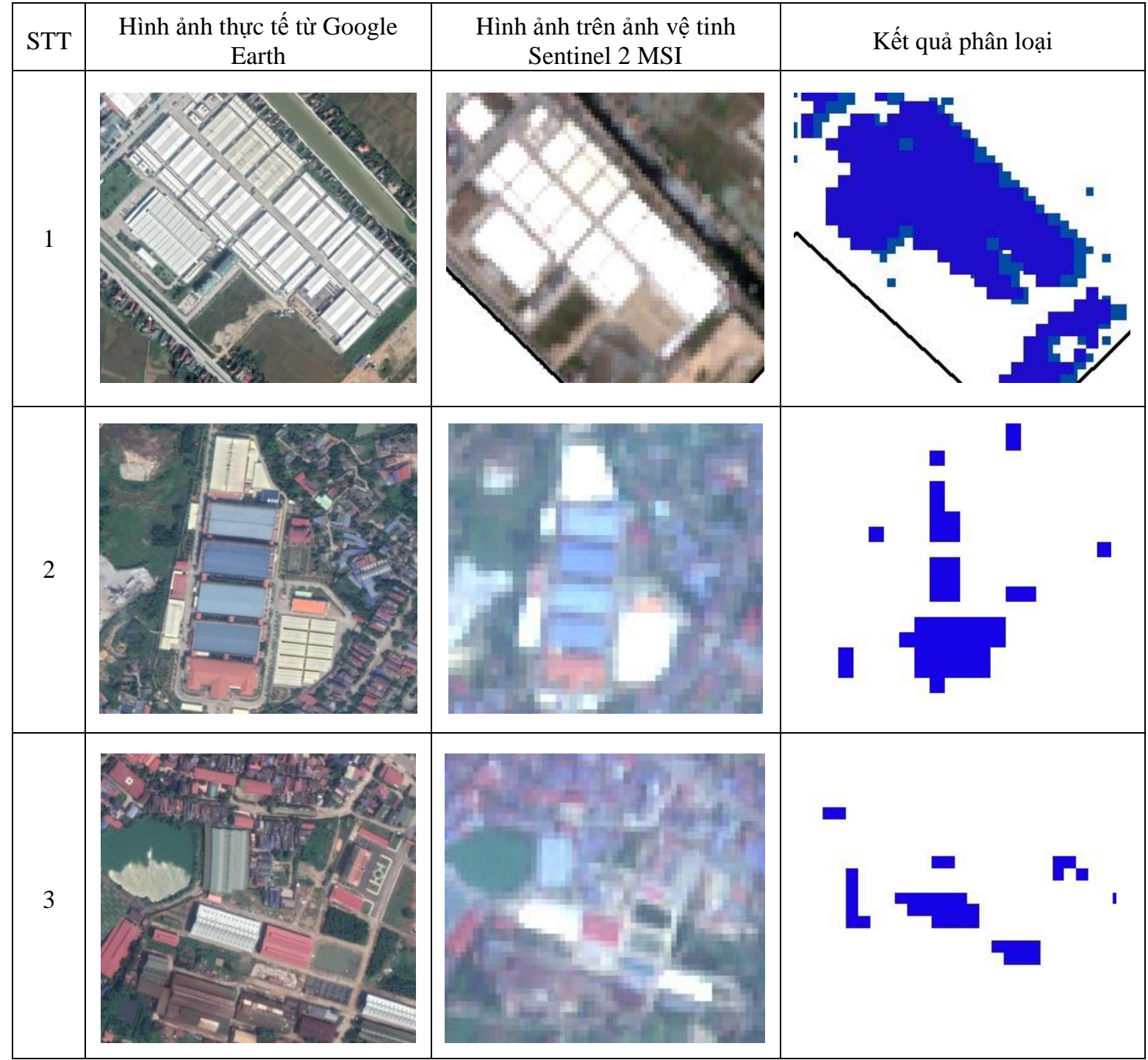

\section{Tài liệu tham khảo}

[1] H.Q. Xu, Spatial expansion of urban/town in Fuqing and its driving analysis, Remote Sensing Technology and Application 17 (2002) 86-92.

[2] J.G. Masek, F.E. Lindsay, S.N. Goward, Dynamics of urban growth in the Washington DC metropolitan area, 1973-1996 from Landsat observations, International Journal of Remote Sensing 21(18) (2000) 3473 - 3486.

[3] B. Guindon, Y. Zhang, C. Dillabaugh, Landsat urban mapping based on a combined spectralspatial methodology, Remote Sensing of Environment 92(2) (2004) 218 - 232.

[4] G. Xian, M. Crane, Assessments of urban growth in the Tampa Bay wateshed using remote sensing 
data, Remote Sensing of Environment 97(2005) 203-215.

[5] M.K. Ridd, Exploring a V-I-S (vegetation-imprevious surface-soil) model for urban ecosystem analysis through remote sensing: comparative anatomy for cities, International Journal of Remote Sensing 16(12) (1994) 2165- 2185.

[6] H.Q. Xu, Extraction of urban built -up land features from Landsat imagery using a thematic oriented index combination technique, Photogrammetric Engineering \& Remote Sensing 73(12) (2007) 1381-1391.

[7] Y. Zha, J. Gao, S. Ni, Use of normalized difference built-up index in automatically mapping urban areas from TM imagery, International Journal of Remote Sensing 24(3) (2003) 583-594.

[8] H.Q. Xu, A study on information extraction of water body with the modified mormalized difference water index (MNDWI), Journal of Remote Sensing 9(5) (2008) 511-517.

[9] N.H.K. Linh, Automatic establishment of urban land distribution map using IBI index from Landsat TM image: Case study in Hue city - Thua Thien Hue province, National Conference on GIS Application (2011) 205-212 (in Vietnamese).

[10] Abd. R. As-syakur, I.W. Adnyana, I.W. Arthana, I.W. Nuarsa, Enhanced built - up and bareness index (EBBI) for mapping built - up and bare land in an urban area, Remote Sensing 4 (2012) 2957 2970.

[11] A. Rasul, H. Balzter, G.I. Faqe, H. Hameed, J. Wheeler, B. Adamu, S. Ibrahim, P. Najmaddin, Applying built-up and bare soil indicies from Landsat 8 to cities in dry climates, Land $7(81)$ (2018) 1-13.

[12] H. Li, C. Wang, C. Zhong, A. Su, C. Xiong, J. Wang, J. Liu, Mapping urban bare land automatically from Landsat imagery with a simple index, Remote Sensing 9(3) (2017) 1-15.

[13] A. Sekertekin, S. Abdikan, A. Marangoz, The acquisition of impervious surface area from
Landsat 8 satellite sensor data using urban indices: a comparative analysis, Environmental Monitoring and Assessment 190(7) (2018) 1-13.

[14] V. Bramhe, S. Ghosh, P. Garg, Extraction of builtup area by combining textural features and spectral indices from Landsat 8 multispectral image, The International Archives of the Photogrammetry, Remote Sensing and Spatial Information Sciences 42(5) (2018) 727-733.

[15] S. Gadal, W. Ouerghemmi, Multi-level morphometric characterization of built-up areas and change detection in Siberian sub-arctic urban area: Yakutsk, International Journal of Geo-Information 8 (2019) 129-149.

[16] H. Zhao, X. Chen, Use of Normalized Difference Bareness Index in quickly mapping bare areas from TM/ETM+, International Geoscience and Remote Sensing Symposium (IGARSS) 3 (2005) 1666-1668. https://doi.org/10.1109/IGARSS.2005. 1526319.

[17] D. Ghosh, A. Mandal, R. Majumder, P. Patra, G. Bhunia, Analysis for mapping of built-up area using remotely sensed indices - A case study of Rajarhat block in Barasat Sanda sub-division in West Bengal (India), Journal of Landscape Ecology 11(2) (2018) 67-76.

[18] M. Firozijaei, A. Sehighi, M. Kiavarz, S. Qureshi, D. Haase, S. Alavipanah, Automated built-up extraction index: a new technique for mapping surface built-up areas using Landsat 8 OLI imagery, Remote Sensing 11 (2019). https://doi. org/10.3390/rs11171966.

[19] E. Mustafa, G. Liu, H. El-Hamid, M. Kaloop, Simulation of land use dynamics and impact on land surface temperature using satellite data, GeoJournal 78(4) (2013) 1-19. https://doi.org/10. 1007/s10708-019-10115-0.

[20] N. Pahlevan, S. Sarkar, B. Franz, S. Balasubramanian, J. He, Sentinel-2 MultiSpectral Instrument (MSI) data processing for aquatic science applications: Demonstrations and validations, Remote Sensing of Environment 201 (2017) 47-56. 